\title{
Computer-aided Glaucoma Diagnosis using Stochastic Watershed Transformation on Single Fundus Images
}

\author{
Andres Diaz-Pinto ${ }^{\mathrm{a}, *}$, Sandra Morales ${ }^{\mathrm{a}}$, Valery Naranjo ${ }^{\mathrm{a}}$, Amparo Navea $^{\mathrm{b}}$ \\ ${ }^{a}$ Instituto de Investigación e Innovación en Bioingeniería, I3B, Universitat Politècnica de València, Camino de Vera s/n, 46022 Valencia, Spain \\ ${ }^{b}$ Instituto de Ciencias Biomédicas, Universidad CEU Cardenal Herrera, Avenida del Seminario s/n, Moncada, 46313 Valencia, Spain
}

\begin{abstract}
Glaucoma is a chronic eye disease and one of the major causes of permanent blindness. Since it does not show initial symptoms, early diagnosis is important to limit its progression. This paper presents an automatic optic nerve characterization algorithm for glaucoma diagnosis based only on retinal fundus images. For optic cup segmentation, we used a new method based on the stochastic watershed transformation applied on the YIQ colour space to extract clinical indicators such as the Cup/Disc ratio, the area Cup/Disc ratio and the ISNT rule. Afterwards, an assessment between normal and glaucomatous fundus images is performed. The proposed algorithm was evaluated on 6 different (private and public) databases containing 723 images (377 normal and 346 glaucomatous images) which achieved a specificity and sensitivity of 0.674 and 0.675 , respectively. Moreover, an F-score of 0.770 was obtained when evaluating this method on the publicly available database Drishti-GS1. A comparison of the proposed work with other state-of-the-art methods demonstrates the robustness of the proposed algorithm; because it was tested using images from different databases with high variability, which is a common issue in this area. Additional comparisons with existing works for cup segmentation, that use the publicly available database Drishti-GS1, are also presented in this paper.
\end{abstract}

Keywords:

Glaucoma; Fundus Images; Stochastic Watershed; CDR; ISNT rule

\section{Introduction}

Glaucoma is the second most prominent eye disease in the world [1]. It is considered as a "multifactorial optic neuropathy" which leads to deterioration in vision and quality of life. Glaucoma is usually asymptomatic and people are commonly unaware of the disease until later stages. For that reason, early detection and treatment are important to prevent vision loss. This silent eye disease is mainly characterized by optic nerve fibre loss and that is given by the increased intraocular pressure (IOP) and/or loss of blood flow to the optic nerve [2]. However, IOP measurement is found to be neither specific nor sensitive enough to be an effective glaucoma indicator since visual damage can be present without increased IOP.

The optic nerve head is physiologically defined as the location where ganglion cell axons leave the eye forming the optic disc (OD). It can be divided into two zones, a central excavation zone called the optic cup and a peripheral part called neuro-retinal rim [3]. See Figure 1.

While the optic disc (OD) and cup are present in all individuals, an abnormal size of the cup with respect to the optic disc is a characteristic of a glaucomatous eye (called "cupping effect") [4]. It can be seen in Figure 2(b). Therefore, a quanti-

\footnotetext{
${ }^{*}$ Corresponding author

Email addresses: andiapin@upv.es (Andres Diaz-Pinto ), sanmomar@upv.es (Sandra Morales), vnaranjo@upv.es (Valery Naranjo), desamparados.navea@uchceu.es (Amparo Navea)
}

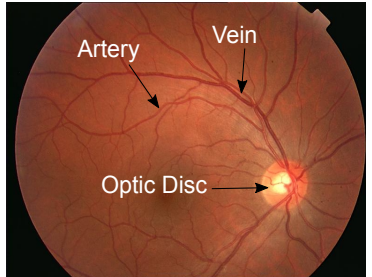

(a)

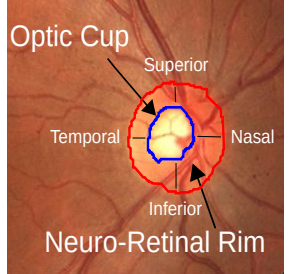

(b)
Figure 1: Digital fundus images. (a) Main structures of an original fundus image and (b) Main structures of the optic disc region.

tative evaluation of the optic nerve can be made by measuring the increased size of the cup using single fundus images.

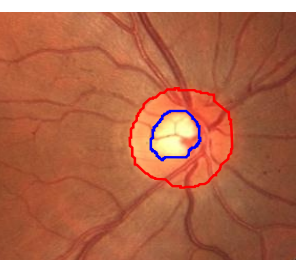

(a)

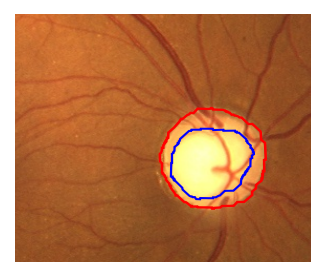

(b)
Figure 2: Visual difference between a healthy and a glaucomatous optic disc: (a) healthy optic disc and (b) glaucomatous optic disc.

There are currently different ways to measure the changes in the cup. A common way is a ratio that expresses the vertical 
diameter proportion of the optic disc and the cup (Cup/Disc ratio or CDR). For normal discs, this proportion falls in the range of 0.3 to 0.5 and is higher than 0.5 for glaucomatous discs [5]. There exist other characteristics such as the ISNT rule and the area Cup/Disc ratio or ACDR. The ISNT rule in a healthy optic disc has a characteristic configuration of the neuro-retinal rim; which is thickest inferiorly, followed by superiorly, then nasally, then temporally ( $>\mathrm{S}>\mathrm{N}>\mathrm{T}$ ) [6]. On the other hand, the ACDR is the ratio between the area occupied by the optic nerve and the area occupied by the cup. Therefore, CDR, ISNT rule and ACDR are quantitative characteristics commonly used for glaucoma assessment and can be gathered manually or by automatic algorithms.

In order to measure CDR, ACDR and ISNT rule, the first step is to segment the optic cup. However, given the optic cup is actually an excavation, representing it as a $2 \mathrm{D}$ object using a colour fundus image makes the automatic cup segmentation process particularly difficult. Different approaches have been developed towards cup segmentation and glaucoma detection using colour fundus images. For instance, Wong et al. and Liu et al. presented a method to calculate the CDR after obtaining the optic cup and optic disc masks using level-set technique [7]. Wong et al. tested their method on 104 images from the Singapore Malay Eye Study, and found that their method produced results with a variation of up to $0.2 \mathrm{CDR}$ units from the ground truth. Another approach for optic disc and optic cup segmentation is presented by Cheng et al. which developed a technique to measure the CDR based on superpixel classification [8]. They evaluated their method on 650 images achieving areas under the ROC curve of 0.800 and 0.822 in two databases. There is also another method proposed by Joshi et al., which is based on anatomical evidence such as vessel bends at the cup boundary. They localised the optic cup using the vessel geometry and circular Hough transform obtaining a CDR error of $0.12 \pm 0.10$ [9]. Other works that use other information such as the patient personal data and patient's genome information is presented in [10]. Different techniques have only been focused on optic disc and/or cup segmentation [11, 12]. In [13], a review of optic disc and cup segmentation methodologies is presented. This review provides a flowchart for each developed technique. It discusses the pros and cons of each optic disc and cup segmentation method.

The main contribution of this work is an automatic algorithm for cup segmentation that is based on the pallor presented in fundus images. Further clinical characteristics such as CDR, ACDR and ISNT rule are measured to finally classify colour fundus images belonging to six different databases with high variability grade. This method is based on the use of Stochastic Watershed transformation. In addition, an extensive analysis of different colour spaces was done to check the best performance in segmenting the cup.

\section{Materials and Methods}

\subsection{Materials}

Four public and two private databases were used in this work. 12 Octubre database which was provided by 12 de Oc- tubre Hospital (Madrid), is composed of 53 annotated images [14]. DRIVE database, composed of 40 images [15]. DrishtiGS1 database, which consists of 101 annotated images by four specialists [12]. HRF database, which contains 45 images [16]. RIM-ONE database, which is composed of 455 images [17], and Autogla database, which is composed of 83 images provided by the FISABIO oftalmología médica (FOM).

Experts of the FISABIO Oftalmología Médica (FOM) analysed the images of the DRIVE database for glaucoma through visual inspection, and based on their analysis they were labelled as Glaucoma or Normal. They also segmented the optic disc and optic cup manually for the images in the HRF, RIMONE and Autogla databases. Examples of these annotations are shown in Figure 3.

It is important to remark that images from RIM-ONE database are originally cropped around the disc and the final ground truth of the Drishti-GS1 database was obtained as the intersection of the ground truth of each specialist. Further to these images, we count with the ground truth of the vessels in HRF and DRIVE databases.

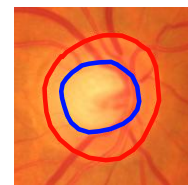

(a)

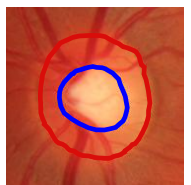

(b)

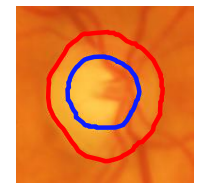

(c)

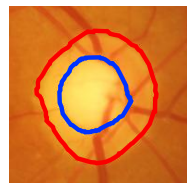

(d)
Figure 3: Annotated images from HRF and Autogla databases. In this figure, images were cropped just for presentation purposes. (a-b) Images from the HRF database. (c-d) Images from the Autogla database.

In total, there were available 777 images belonging to 6 databases. However, experiments were only carried out in images with a minimum quality. Based on specialists' criteria, images with doubtful diagnosis, low resolution or images which optic cup is not discernible were excluded from the experiment. Examples of them are shown in Figure 4.

Therefore, a total of 723 images were used for the experiments. 346 were related to glaucomatous and 377 to healthy images, as it is shown in Table 1.

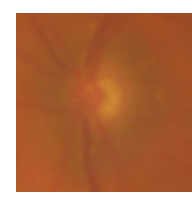

(a)

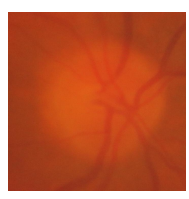

(b)

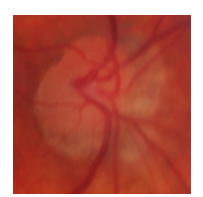

(c)

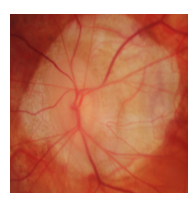

(d)
Figure 4: Different samples of the excluded images. (a) Image with low resolution, (b-c) Examples of images which optic cup is not discernible and (d) Image with a doubtful diagnosis.

\subsection{Colour Spaces}

A fundus camera captures a retinal image in the RGB colour space (Red, Green and Blue). However, depending on the anatomical or pathological structure of the retina to be analysed, a component of certain colour space could provide more 
Table 1: Number of images in each database used for the experiments

\begin{tabular}{lccc}
\hline Name & Number of images & Glaucoma & Normal \\
\hline 12Octubre [14] & 53 & 29 & 24 \\
Drive [15] & 40 & 20 & 20 \\
Drishti-GS1 [12] & 101 & 70 & 31 \\
Autogla & 83 & 50 & 33 \\
HRF [16] & 45 & 27 & 18 \\
RIM-ONE [17] & 401 & 150 & 251 \\
Total & $\mathbf{7 2 3}$ & $\mathbf{3 4 6}$ & $\mathbf{3 7 7}$ \\
\hline
\end{tabular}

information than others. For instance, for vessel segmentation, the green channel in the RGB representation is widely used in the literature [18]. In this work, the RGB and five more colour spaces were analysed in order to determine which one performs better for cup segmentation using the Stochastic Watershed transformation. The other five colour spaces are the Lab and Luv (created by the International Commission on Illumination (CIE) [19], the CMYK (Cyan, Magenta, Yellow, and Key (black) components) colour model, the PCA (Principal Component Analysis) [20], and the YIQ (Used in NTSC colour TV system).

\subsection{Stochastic Watershed}

As it was mentioned above, the proposed algorithm uses the Stochastic Watershed transformation to segment the optic cup. Based on the gradient image, Stochastic Watershed transformation is a segmentation technique in which the minimum pixel values of the image represent the object of interest and the maximum pixel values represent the separation boundaries between objects [21].

One problem with the watershed technique is the oversegmentation, which is caused by the existence of numerous local minima in the image due to the presence of noise. This problem is solved with the marker-controlled watershed, establishing the image minima artificially and defining a marker per minimum. Internal and external markers are needed. The internal markers determine the object of interest and the external markers are used to limit the segmented area. In this work, the contour of the optic disc, which was obtained from the method proposed in [11], was used as the external marker. Internal markers are regionalized random markers whose distribution is restricted to areas that accomplished a specific condition. In this case, they must be located in low-intensity or high-intensity areas depending on the colour space. When the colour spaces CMYK, Lab, Luv and YIQ are used, the cup is the darkest part of the optic disc and when the RGB and PCA colour space is used, the cup is the brightest part of the optic disc. Starting with an initial marker or seed which is defined by the darkest or the brightest pixel, the intensity regionalized markers or internal markers are chosen by following a Poisson distribution with variance $\sigma^{2}$ [22].

In particular, in Stochastic Watershed transformation, a given number $M$ of marker-controlled watershed realizations are performed selecting $N$ regionalized random markers (or pseudorandom markers) in each realization. The idea is to estimate a probability density function ( $p d f$ ) for the countours of the image, which filter out non-significant border fluctuations. The probability density function is computed by Parzen window method [23] as: $p d f(\mathbf{x})=1 / M * \sum_{i=1}^{M}\left(W S_{i}(\mathbf{x}) * G(\mathbf{x} ; s)\right)$, where $G(\mathbf{x} ; s)$ represents a Gaussian function of variance $s^{2}$ and mean $\mu(\mu=0), M$ represents the number of marker-controlled watershed realizations with $N$ regionalized random markers and $W S_{i}=W S(\varrho)_{f_{m k} k_{i}}$ the $i$ th output watershed image, being $\varrho$ the gradient image. Afterwards, it is necessary to perform a last marker-controlled watershed transformation on the $p d f$, which defines the resulting mask by joining all the watershed regions.

In Section 3.2 the intermediate and final result of the Stochastic Watershed algorithm for optic cup segmentation will be shown.

\section{Proposed Method}

The automatic algorithm proposed in this paper is mainly focused on using the Stochastic Watershed transformation for the cup segmentation, and then, measuring the CDR, ACDR and the ISNT rule to classify fundus images into normal and glaucomatous.

For cup segmentation, a preprocessing of the original retinal images is required. First, the optic disc mask for each image was obtained automatically by using the method proposed in [11]. This method also uses the same Stochastic Watershed transformation to segment the optic disc. Secondly, images are resized and cropped around the optic disc. Thirdly, grey-scale transformation and vessel removal are necessary steps previous to finally apply the Stochastic Watershed transformation.

The flowchart of the proposed method is presented in Figure 5 and described below.

\subsection{Preprocessing}

\subsubsection{Image resize}

Image resize is the first part of the preprocessing block. Due to the fact that the images under study belong to different databases, the image size varies. This block resizes the images to a standardized size in order to obtain comparable results between the databases. In this work, the 12Octubre's image size was used $(768 \times 576)$ because is the lowest resolution in all databases.

To resize the images, the length of the horizontal diameter of the fundus was used as reference [24]. Bicubic interpolation was used for resizing; in which the output pixel value is a weighted average of pixels in the nearest 4-by-4 neighbourhood.

\subsubsection{ROI localization}

After image resizing, the next step is to crop the images strategically. The proposed method crops the original image taking as reference the optic disc with about 100 more pixels around it. An example of a cropped image is shown in Figure 6(a). 


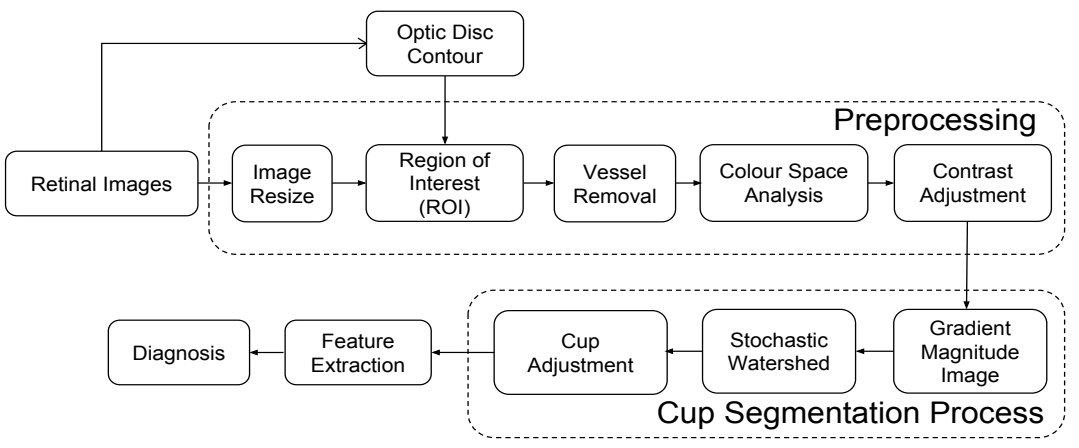

Figure 5: Complete block diagram of the proposed algorithm

\subsubsection{Vessel removal}

The glaucoma disease is mainly characterized by the loss of the optic nerve and astrocytes. However, to the best of our knowledge, the vessels are not directly affected by this disease. For that reason, an important preprocessing step is to remove the vessels which do not provide extra information for glaucoma diagnosis and can make the cup segmentation more difficult.

Only the HRF and the DRIVE database were provided with the vessel masks. For the other databases (12Octubre, DrishtiGS1, Autogla and RIM-ONE), the segmentation of the vessels was needed. To do that, a method based on mathematical morphology, curvature evaluation and the k-means clustering algorithm was used [25]. Afterwards, an iterative inpainting technique [26], used in photo restoration and video processing, replaces the pixels of the vessel mask using exemplar-based synthesis.

\subsubsection{Colour space analysis}

After images are resized, cropped and without vessels, the components of the colour spaces CMYK, PCA, YIQ, Lab, Luv and RGB were analysed. The reason for this analysis is to check the performance of each component in cup segmentation and choose that which provides optimal results.

\subsubsection{Contrast adjustment}

It was observed that the nonuniform-contrast image through the available databases affects the classification performance. For this reason, a contrast adjustment is applied on the greyscale image obtained after colour space decomposition. After obtained the grey-scale image using the colour space model, the intensity values in that image are linearly mapped into another grey-scale image such that $1 \%$ of data is saturated at low and high intensities, with the aim of obtaining a higher difference between the intensity values in the cup and the rest of the image.

\subsection{Cup segmentation}

The cup segmentation block is mainly divided into 2 parts: the Stochastic Watershed transformation and the Cup adjustment block.

\subsubsection{Stochastic Watershed Segmentation}

Fundamentally, this is the neuralgic step in the proposed method. As it was previously mentioned, the Stochastic Watershed transformation makes $M$ marker-controlled watershed realizations with $N$ pseudorandom markers to finally obtain a $p d f$. Therefore, the variable parameters of the watershed algorithm were carefully set (the variance of the Poisson function that generates the random markers, the number of random markers and the number of realizations). Given the small intensity variance between the pixels in the cup, the variance $\sigma^{2}$ was set experimentally in a small value $\left(\sigma^{2}=0.0003\right)$. The algorithm was programmed to generate a minimum random markers (N) of 100. This means if the initial variance is not enough to generate a 100 random markers, the variance values increases until there are at least the minimum number of markers. Another important factor is the number of realizations (M). It was observed that 5 realizations are a good trade-off between a suitable $p d f$ for segmentation and a relatively low computational cost. For that reason, this factor was set at 5 .

Given that the markers are limited by the region of interest of the image, they are not completely random. This limitation makes them pseudo-random markers.

\subsubsection{Cup adjustment}

The cup adjustment block improves the measure of the Cup/Disc ratio and ISNT rule. In this block, the vertical radius of the mask obtained by the watershed transformation is calculated, then a circle is generated with the same vertical radius of the watershed mask and placed in the centre of the optic disc. The main motivation for this adjustment came from the fact that the temporal part of the optic cup is usually hidden by the vessels [5]. The reason for placing this circle in the centre of the optic disc is because the optic cup is generally located in the centre of the optic disc.

In Figure 6(j) an example of the cup adjustment result is shown. The black line represents the segmentation using the proposed method based on Stochastic Watershed, the white line represents the adjustment made to the segmented cup and the yellow and blue lines identify the ground truth of the optic disc and cup, respectively. 


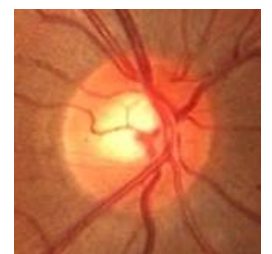

(a)

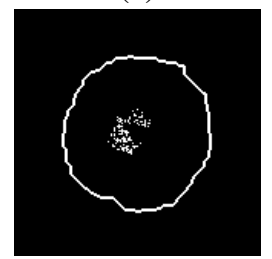

(f)

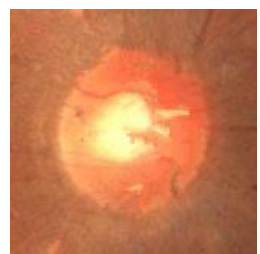

(b)

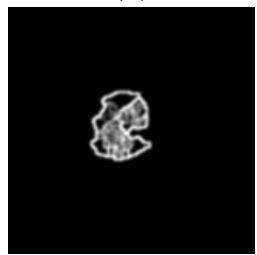

(g)

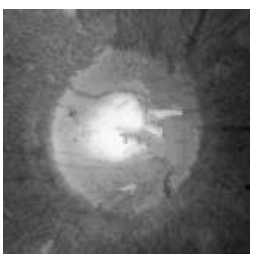

(c)

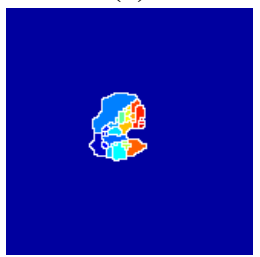

(h)

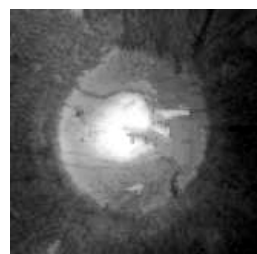

(d)

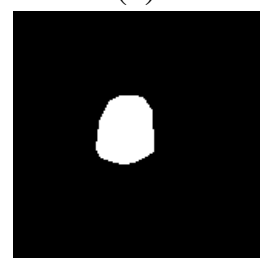

(i)

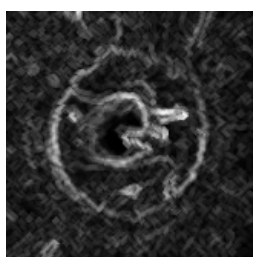

(e)

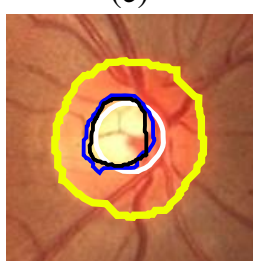

(j)

Figure 6: Process of the Stochastic Watershed transformation: (a) Resized and cropped image (ROI), (b) Image inpainting, (c) Grey-scale image (first PCA component), (d) Contrast adjust, (e) Gradient image, (f) Pseudo-random markers, (g) pdf of image contours, (h) Watershed regions, (i) Final segmentation and (j) Cup adjustment (in white).

\subsection{Glaucoma diagnosis}

At this point, cup segmentation process using Stochastic Watershed has been shown. Next step is to use the obtained optic cup mask to measure the CDR, ACDR and the ISNT rule and make a glaucoma assessment.

ACDR is defined as the ratio between the area occupied by the cup and the area occupied by the optic disc. CDR is the ratio of the vertical diameter of the cup and the vertical diameter of the optic disc [6]. The proposed algorithm measures the CDR by computing the diameter of the optic disc and the cup, as the mean of the two highest vertical diameters because of the irregular shape of the optic disc and the cup.

Optimum thresholds for glaucoma diagnosis were stablished to $\mathbf{0 . 5 0}$ for the CDR and $\mathbf{0 . 3 0}$ for ACDR [5].

Regarding the ISNT rule, the horizontal and vertical thickness of the neuro-retinal rim were measured as it was done with the CDR (by mean of the two highest values). The horizontal thickness represents the temporal part (T) plus the nasal part (N) of the neuro-retinal rim: HorDiam $=T+N$, and the vertical thickness represents the inferior part (I) plus the superior part $(\mathrm{S})$ of the neuro-retinal rim: VerDiam $=I+S$. If the horizontal thickness is smaller than the vertical thickness, the optic disc in the image follows the ISNT rule, and then, classified as "Normal", otherwise it is classified as "Glaucomatous".

\section{Results and Discussion}

As it was mentioned in Section 2.1, 723 images belonging to 6 different databases were used for this work.

\subsection{Cup segmentation}

In order to test the performance of the cup segmentation, the Jaccard and Dice indexes were calculated for every image, and the mean and standard deviation were obtained for each database. After that, the weighted average and standard deviation of the Jaccard and Dice indexes were calculated for all databases. This means they are calculated according to the number of images of each database. This analysis was performed for all the six colour spaces (CMYK, PCA, YIQ, Lab, Luv and RGB).

As it can be seen from Table 2, HRF and RIM-ONE databases have better cup segmentation results when YIQ colour space is used. Given these databases have the majority of the images (446/723 images), the weighted average is higher when YIQ colour space is applied. However, the results obtained when using the CMYK colour space, have also a considerable performance in cup segmentation.

In most cases, using the Stochastic Watershed transformation a proper contour of the optic cup is obtained. Examples of these results can be observed in Figure 7(a-b). However, wrong contours are obtained when the optic cup has not its characteristic pallor. For that reason, a weakness of this method lies in the pallor absence of the optic cup. Which makes the optic cup not discernible from other parts of the optic disc. Examples of this problem can be seen in Figure 7(c-d).

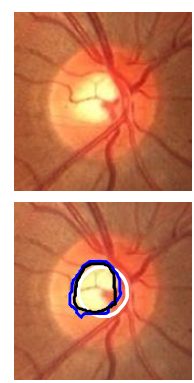

(a)
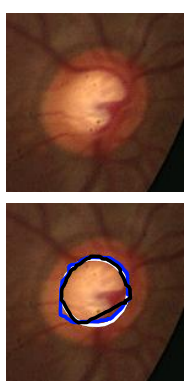

(b)
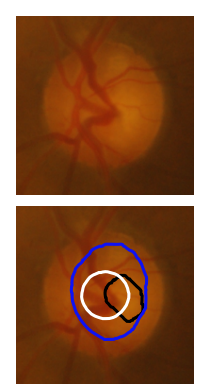

(c)
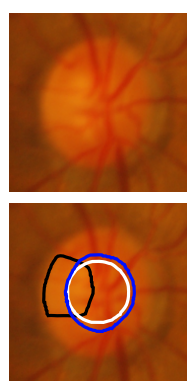

(d)
Figure 7: Results of best and worst cases of the optic cup segmentation (upper images are the original images cropped around the optic disc). The blue line represents the annotation made by the experts, the black line represents the contour obtained from the Stochastic Watershed transformation and the white line represents the cup adjustment.

In order to compare the proposed method with other state-ofthe-art methods, the overlapping error $E$ was computed. This evaluation metric is defined as $E=1-\operatorname{Area}(S \cap G) / \operatorname{Area}(S \cup G)$, 
Table 2: Results of the cup segmentation using six colour spaces.

\begin{tabular}{cccccccc}
\hline Database & Index & CMYK & YIQ & Luv & Lab & PCA & RGB \\
\hline \multirow{2}{*}{ 12Octubre } & Jaccard & $0,553 \pm 0,185$ & $0,565 \pm 0,215$ & $0,526 \pm 0,260$ & $0,551 \pm 0,238$ & $0,558 \pm 0,214$ & $\mathbf{0 , 5 7 5} \pm \mathbf{0 . 1 9 2}$ \\
& Dice & $0,692 \pm 0,173$ & $0,695 \pm 0,204$ & $0,643 \pm 0,281$ & $0,674 \pm 0,250$ & $0,689 \pm 0,202$ & $\mathbf{0 , 7 0 9} \pm \mathbf{0 . 1 7 8}$ \\
\hline \multirow{2}{*}{ DRIVE } & Jaccard & $\mathbf{0 , 5 6 7} \pm \mathbf{0 , 1 9 8}$ & $0,481 \pm 0,227$ & $0,415 \pm 0,247$ & $0,461 \pm 0,224$ & $0,471 \pm 0,211$ & $0,495 \pm 0.200$ \\
& Dice & $\mathbf{0 , 7 0 0} \pm \mathbf{0 , 1 9 1}$ & $0,615 \pm 0,233$ & $0,541 \pm 0,269$ & $0,596 \pm 0,239$ & $0,611 \pm 0,209$ & $0,638 \pm 0.187$ \\
\hline \multirow{2}{*}{ Drishti-GS1 1} & Jaccard & $0,656 \pm 0.191$ & $0,661 \pm 0.215$ & $0,347 \pm 0.350$ & $0,492 \pm 0.328$ & $\mathbf{0 , 6 6 8} \pm \mathbf{0 . 2 0 0}$ & $0,664 \pm 0.185$ \\
& Dice & $0,775 \pm 0.160$ & $0,771 \pm 0.190$ & $0,416 \pm 0.391$ & $0,583 \pm 0.356$ & $\mathbf{0 , 7 8 1} \pm \mathbf{0 . 1 7 5}$ & $0,780 \pm 0.161$ \\
\hline \multirow{2}{*}{ Autogla } & Jaccard & $0,568 \pm 0,197$ & $0,551 \pm 0,210$ & $0,512 \pm 0,270$ & $0,527 \pm 0,240$ & $0,560 \pm 0,209$ & $\mathbf{0 , 5 7 2} \pm \mathbf{0 . 2 0 1}$ \\
& Dice & $0,702 \pm 0,177$ & $0,683 \pm 0,207$ & $0,626 \pm 0,296$ & $0,653 \pm 0,245$ & $0,693 \pm 0,194$ & $\mathbf{0 , 7 0 6} \pm \mathbf{0 . 1 8 0}$ \\
\hline \multirow{2}{*}{ HRF } & Jaccard & $0,607 \pm 0,185$ & $\mathbf{0 , 6 2 7} \pm \mathbf{0 , 1 5 0}$ & $0,545 \pm 0,250$ & $0,592 \pm 0,221$ & $0,575 \pm 0,212$ & $0,614 \pm 0.179$ \\
& Dice & $0,738 \pm 0,161$ & $\mathbf{0 , 7 5 9} \pm \mathbf{0 , 1 3 1}$ & $0,663 \pm 0,269$ & $0,712 \pm 0,234$ & $0,704 \pm 0,199$ & $0,744 \pm 0.161$ \\
\hline \multirow{2}{*}{ RIM-ONE } & Jaccard & $0,523 \pm 0,190$ & $\mathbf{0 , 5 4 0} \pm \mathbf{0 , 1 7 8}$ & $0,329 \pm 0,287$ & $0,464 \pm 0,253$ & $0,491 \pm 0,209$ & $0,515 \pm 0.198$ \\
& Dice & $0,665 \pm 0,175$ & $\mathbf{0 , 6 8 3} \pm \mathbf{0 , 1 6 9}$ & $0,423 \pm 0,336$ & $0,586 \pm 0,281$ & $0,630 \pm 0,202$ & $0,656 \pm 0.185$ \\
\hline \multirow{2}{*}{ All databases } & Jaccard & $0,559 \pm 0,203$ & $\mathbf{0 , 5 6 5} \pm \mathbf{0 , 2 1 8}$ & $0,546 \pm 0,259$ & $0,468 \pm 0,233$ & $0,546 \pm 0,227$ & $0.537 \pm 0.205$ \\
& Dice & $0,696 \pm 0,187$ & $\mathbf{0 , 7 0 2} \pm \mathbf{0 , 2 2 1}$ & $0,679 \pm 0,281$ & $0,590 \pm 0,226$ & $0,682 \pm 0,215$ & $0.674 \pm 0.190$ \\
\hline
\end{tabular}

where $S$ and $G$ denote the segmented cup and the ground truth respectively.

Table 3 shows the percentage images per interval of overlapping error and the average $\mu_{E}$ using the annotated images as ground truth. The results are shown in detail for each database, and for all the images combined.

It can be seen from the table that the percentage of images for the Drishti-GS1 database has better relative results compare to the other databases. However, the RIM-ONE database has more impact on the final result because of the number of images.

For the comparison, the results presented in [8] were used. Those results were obtained from experiments using cup segmentation algorithms such as the threshold method [9], r-bend [27], ASM method [28] and regression method [29].

Table 4 gives a comparative analysis of the performance of the proposed method against some existing methods of cup segmentation. It is possible to see from the table that the proposed algorithm is among the methods with the best performance. In addition, this method is evaluated with a greater number of images.

Unlike existing works, the proposed method uses databases from different hospitals and research centres, which implies different image resolution and image quality, thus it demonstrates the robustness of the method in case of variability conditions. For instance, Regression and Superpixel methods were evaluated using fewer images and from only one private database.

Another comparison with works that only use the DrishtiGS1 database was made. For this comparison the F-score, which is defined as $F=2 P \times R /(P+R)$ was calculated, where $P$ is precision and $R$ is the recall. For instance, Sedai et al. [31] obtained an F-score of 0.86 using only 50 of the 101 images in the Drishti-GS1 database and Chakravarty et al. [32] obtained an F-score of 0.81 using the whole Drishti-GS1 database. As in the latter, in this work, all the images in the Drishti-GS1 were used; obtaining an F-score of 0.77. It shows that the proposed algorithm has a competitive performance for optic cup segmentation.

\subsection{Glaucoma diagnosis}

After the cup is segmented, the CDR, ACDR and ISNT rule was measured for all the images. Table 5 shows the specificity (Sp) and sensitivity (Se) obtained for each colour space that was used in the cup segmentation process.

It can be observed from Table 2 that YIQ colour space presents better performance overall the other colour spaces in cup segmentation. It is also possible to see from Table 5, that measuring the CDR using the colour space YIQ, the glaucoma diagnosis is more reliable than measuring the ACDR, the ISNT rule or combining all of them. For that reason, next results were obtained using the YIQ colour space and measuring the CDR.

In Figure 8, the ROC curves for each database can be observed. In these figures, blue lines represent the ROC curves for the ground truth and the red lines represent the obtained results from the proposed method measuring the CDR and using the YIQ colour space. It was observed from the experiments that the ROC plot for the Drishti-GS1 database has the best relative result compared to the other databases.

After the ROC curves for each database were generated, a weighted average ROC curve was computed using the results of all databases. In Figure 9(a) is possible to see a comparison between the ground truth and the results of the proposed method. And in Figure 9(b), a comparison between the superpixel results, using SiMES database [8], and the results of the proposed method. It is important to remark that the superpixel method and the proposed method were tested with different databases. It can be seen that although the experiments were made with a wide variety of images, the proposed method performs properly and its results can be considered clinically significant.

\subsection{Computation time}

Our whole method was implemented in MatLab on a 3.40 $\mathrm{GHz}$ quad-core CPU running Windows 8.1. Once the Stochastic Watershed parameters were tuned an average of $0.508 \mathrm{~s}$ per image were needed to segment, adjust the optic cup contour and measured the CDR, ACDR and ISNT rule. This time 
Table 3: Percentage of images per overlapping error $E$ interval and the average $\mu_{E}$ for cup segmentation purpose.

\begin{tabular}{lccccccc}
\hline & $\#$ images & $E<=0,1$ & $E<=0,2$ & $E<=0,3$ & $E<=0,4$ & $E<=0,5$ & $\mu_{E}$ \\
\hline 12Octubre & 53 & $1,89 \%$ & $15,09 \%$ & $39,62 \%$ & $62,26 \%$ & $69,81 \%$ & $38,60 \%$ \\
DRIVE & 40 & $0 \%$ & $12,50 \%$ & $27,50 \%$ & $52,50 \%$ & $75 \%$ & $41,40 \%$ \\
Drishti-GS1 & 101 & $\mathbf{6} \%$ & $\mathbf{3 4} \%$ & $\mathbf{6 4} \%$ & $\mathbf{6 8} \%$ & $\mathbf{8 6} \%$ & $\mathbf{3 0 , 8 5} \%$ \\
Autogla & 83 & $0 \%$ & $9,64 \%$ & $37,35 \%$ & $56,63 \%$ & $71,08 \%$ & $40,42 \%$ \\
HRF & 45 & $0 \%$ & $17,78 \%$ & $40 \%$ & $71,11 \%$ & $86,67 \%$ & $35,09 \%$ \\
RIM-ONE & 401 & $0,25 \%$ & $5,74 \%$ & $22,44 \%$ & $42,14 \%$ & $61,60 \%$ & $44,72 \%$ \\
\hline All databases & 723 & $\mathbf{1 . 1 1} \%$ & $\mathbf{1 1 . 9 4} \%$ & $\mathbf{3 2 . 5 9} \%$ & $\mathbf{5 1 . 2 7} \%$ & $\mathbf{6 9 . 0 0} \%$ & $\mathbf{4 1 . 0 6} \%$ \\
\hline
\end{tabular}

Table 4: Comparison between existing methods and the proposed method using the overlapping error $E$ in cup segmentation. Other works results shown in this table were taken from [8]

\begin{tabular}{lccccccc}
\hline & $E<=0,1$ & $E<=0,2$ & $E<=0,3$ & $E<=0,4$ & $E<=0,5$ & $\mu_{E}$ & Images used \\
\hline Thresholding [9] & $0 \%$ & $3 \%$ & $15 \%$ & $31 \%$ & $47 \%$ & $53,50 \%$ & 138 \\
R-bend [27] & $0 \%$ & $4 \%$ & $28 \%$ & $56 \%$ & $77 \%$ & $39,50 \%$ & 200 \\
Proposed method & $\mathbf{1} \%$ & $\mathbf{1 0} \%$ & $\mathbf{3 0} \%$ & $\mathbf{5 0} \%$ & $\mathbf{6 9} \%$ & $\mathbf{4 1 . 0 6} \%$ & $\mathbf{7 2 3}$ \\
ASM [28] & $3 \%$ & $25 \%$ & $51 \%$ & $76 \%$ & $88 \%$ & $31,30 \%$ & 325 \\
Regression [29] [30] & $6 \%$ & $29 \%$ & $62 \%$ & $81 \%$ & $95 \%$ & $28,40 \%$ & 650 \\
Superpixel [8] & $8 \%$ & $42 \%$ & $75 \%$ & $90 \%$ & $96 \%$ & $24,10 \%$ & 650 \\
\hline
\end{tabular}

Table 5: Results of the Glaucoma diagnosis using the CDR, ACDR, ISNT and the combination of all of them (Combined). In this table, Sp stands for Specificity and Se stands for Sensitivity

\begin{tabular}{|c|c|c|c|c|c|c|c|c|c|c|c|c|}
\hline & \multicolumn{2}{|c|}{ CMYK } & \multicolumn{2}{|c|}{ YIQ } & \multicolumn{2}{|c|}{ Luv } & \multicolumn{2}{|c|}{$\mathrm{Lab}$} & \multicolumn{2}{|c|}{ PCA } & \multicolumn{2}{|c|}{ RGB } \\
\hline & $\mathrm{Sp}$ & $\mathrm{Se}$ & Sp & $\mathrm{Se}$ & Sp & $\mathrm{Se}$ & $\mathrm{Sp}$ & $\mathrm{Se}$ & Sp & $\mathrm{Se}$ & Sp & $\mathrm{Se}$ \\
\hline CDR & 0,574 & 0,697 & 0,675 & 0,674 & 0,650 & 0,731 & 0,832 & 0,563 & 0,487 & 0,716 & 0.545 & 0.716 \\
\hline ACDR & 0,601 & 0,633 & 0,715 & 0,604 & 0,688 & 0,673 & 0,849 & 0,509 & 0,517 & 0,663 & 0.574 & 0.655 \\
\hline ISNT & 0,495 & 0,570 & 0,431 & 0,568 & 0,422 & 0,561 & 0,337 & 0,609 & 0,523 & 0,544 & 0.499 & 0.511 \\
\hline Combined & 0,545 & 0,702 & 0,730 & 0,602 & 0,685 & 0,635 & 0,373 & 0,760 & 0,376 & 0,778 & 0.513 & 0.742 \\
\hline
\end{tabular}

clearly highlights a strength of this method, its computational efficiency.

\section{Conclusions}

An automatic algorithm for optic cup segmentation and feature extraction to further classify glaucomatous images was presented. A sensitivity of 0.675 and a specificity of 0.674 were obtained from the proposed algorithm using the YIQ colour space and measuring the CDR. The segmentation of the optic cup is based on the Stochastic Watershed transformation. This segmentation and glaucoma-healthy classification were validated on 723 colour fundus images from six different databases with high variability. An F-score of 0.770 was obtained when evaluating this method on the publicly available database Drishti-GS1.

\section{Acknowledgements}

The authors would like to thank K. Narasimhan from SASTRA University for facilitating access to their database and gratefully acknowledge the support of NVIDIA Corporation with the donation of the Titan V GPU used for this research.

This work was supported by the Project GALAHAD [H2020-ICT-2016-2017, 732613]. In particular, the work of Andres Diaz-Pinto has been supported by the Generalitat Valenciana under the scholarship Santiago Grisolía [GRISOLIA/2015/027].

\section{References}

[1] Bulletin of the World Health Organization, Volume 82, Number 11, http://www. who.int/bulletin/volumes/82/11/en/infocus . pdf?ua=1, 2004 [accessed 05 May 2017].

[2] R. N. Weinreb, P. T. Khaw, Primary open-angle glaucoma, The Lancet 363 (9422) (2004) 1711-1720. doi:10.1016/S0140-6736(04)16257-0.

[3] R. N. Weinreb, T. Aung, F. A. Medeiros, The Pathophysiology and Treatment of Glaucoma: A Review, JAMA 311 (18) (2014) 1901-1911. doi:10.1001/jama.2014.3192.

[4] Y. H. Kwon, J. H. Fingert, M. H. Kuehn, W. L. Alward, Primary openangle glaucoma, New England Journal of Medicine 360 (11) (2009) 1113-1124. doi:10.1056/NEJMra0804630.

[5] M. K. Nath, S. Dandapat, Techniques of glaucoma detection from color fundus images: A review, International Journal of Image, Graphics and Signal Processing. 4 (2012) 44-51. doi:10.5815/ijigsp.2012.09.07. 

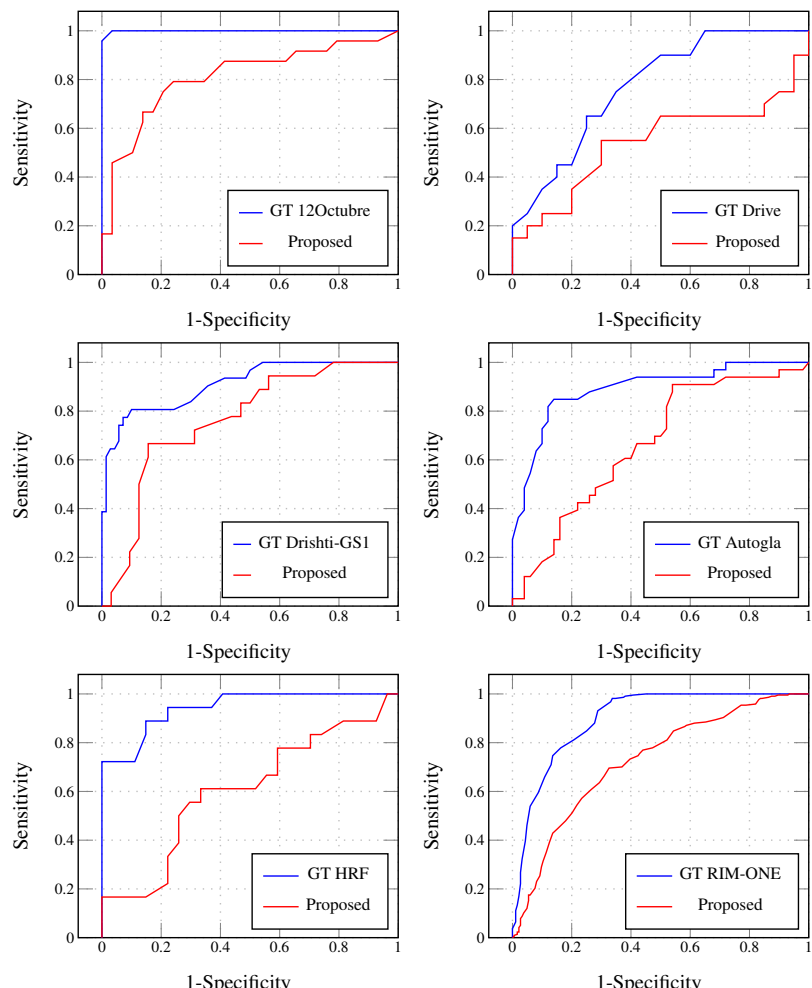

Figure 8: Receiver operating characteristic (ROC) curves for the automatic glaucoma diagnosis for each database using the ground truth (GT) images and the results of the proposed method using the YIQ colour space and the CDR.
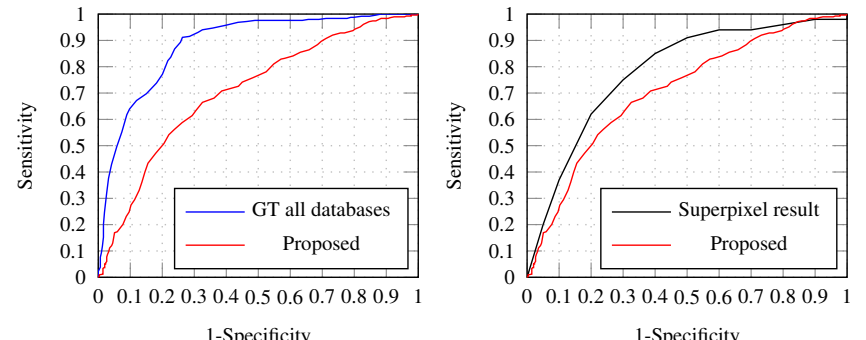

Figure 9: A comparison ROC plots between the weighted average ROC curves for all databases using the ground truth (GT) images and the proposed method (upper) and the Superpixel method and the proposed method (lower).

[6] optic-disc.org, an online resource for ophthalmologists, physicians, medical students and optometrists. glaucoma evaluation tutorial, http://www.optic-disc.org/tutorials/glaucomal _evaluation\_basics/page13.html 2010 [accessed 27 May 2017].

[7] D. W. K. Wong, J. Liu, J. Lim, X. Jia, F. Yin, H. Li, T. Y. Wong, Levelset based automatic cup-to-disc ratio determination using retinal fundus images in ARGALI, 30th Annual International IEEE EMBS Conference. 30 (2008) 2266-2269. doi:10.1109/IEMBS.2008.4649648.

[8] J. Cheng, J. Liu, Y. Xu, F. Yin, D. W. K. Wong, N.-M. Tan, D. Tao, C.-Y. Cheng, T. Aung, T. Y. Wong., Superpixel classification based optic disc and optic cup segmentation for glaucoma screening, IEEE Transactions on Medical Imaging. 32 (2013) 1019-1032. doi:10.1109/TMI.2013.2247770.

[9] G. D. Joshi, J. Sivaswamy, S. R. Krishnadas, Optic disk and cup segmentation from monocular color retinal images for glaucoma assessment, IEEE Transactions on Medical Imaging. 30 (2011) 1192-1205. doi:10.1109/TMI.2011.2106509.
[10] J. Liu, Z. Zhang, D. W. K. Wong, Y. Xu, F. Yin, J. Cheng, N. M. Tan, C. K. Kwoh, D. Xu, Y. C. Tham, T. Aung, T. Y. Wong, Automatic glaucoma diagnosis through medical imaging informatics, Journal of the American Medical Informatics Association. 1 (2013) 1021-1027. doi:U10.1136/amiajnl-2012-001336.

[11] S. Morales, V. Naranjo, J. Angulo, M. Alcañiz, Automatic detection of optic disc based on PCA and mathematical morphology, IEEE Transactions on Medical Imaging. 32 (2013) 786-796. doi:10.1109/TMI.2013.2238244

[12] J. Sivaswamy, S. Krishnadas, G. D. Joshi, M. Jain, Ujjwal, S. T. A., Drishti-GS: Retinal image dataset for optic nerve head $(\mathrm{ONH})$ segmentation, 2014 IEEE 11th International Symposium on Biomedical Imaging (ISBI). 11 (2014) 53-56. doi:10.1109/ISBI.2014.6867807.

[13] A. Almazroa, R. Burman, K. Raahemifar, V. Lakshminarayanan, Optic Disc and Optic Cup Segmentation Methodologies for Glaucoma Image Detection: A Survey, Hindawi Publishing Corporation. Journal of Ophthalmology. 2015 (2015) 28. doi:10.1155/2015/180972.

[14] R. Román Morán, R. Barea Navarro, L. Boquete Vázquez, E. López Guillén, J. Campos Pavón, L. de Pablo Gómez de Liaño, D. Escot Bocanegra, L. de Santiago, M. Ortiz, Color analysis in Retinography: Glaucoma Image Detection, XIII Mediterranean Conference on Medical and Biological Engineering and Computing 2013. 41 (2014) 325-329. doi:10.1007/978-3-319-00846-2_81.

[15] Drive: Digital retinal images for vessel extraction, http://www.isi . uu.nl/Research/Databases/DRIVE/, 2004 [accessed 14 May 2017].

[16] T. Köhler, A. Budai, M. F. Kraus, J. Odstrčilik, G. Michelson, J. Hornegger, Automatic no-reference quality assessment for retinal fundus images using vessel segmentation, Proceedings of the 26th IEEE International Symposium on Computer-Based Medical Systems. 26 (2013) 95-100. doi:10.1109/CBMS.2013.6627771.

[17] E. Medina-Mesa, M. Gonzalez-Hernandez, J. Sigut, F. Fumero-Batista, C. Pena-Betancor, S. Alayon, M. G. de la Rosa, Estimating the amount of hemoglobin in the neuroretinal rim using color images and OCT, Current Eye Research 41 (2015) 798-805. doi:10.3109/02713683.2015.1062112.

[18] D. Singh, Dharmveer, B. Singh, A new morphology based approach for blood vessel segmentation in retinal images (2014) 16doi:10.1109/INDICON.2014.7030686.

[19] H. Mustroph, Dyes, General Survey, Wiley-VCH Verlag GmbH \& Co, 2014. doi:10.1002/14356007.a09_073.pub2.

[20] K. Pearson, On lines and planes of closest fit to systems of points in space, Philosophical Magazine. 2 (1901) 559-572. doi:10.1080/14786440109462720.

[21] S. Beucher, F. Meyer, The morphological approach to segmentation: The watershed transformation, New York: Marcel Dekker, 1992, Ch. 12.

[22] J. Angulo, D. Jeulin, Stochastic watershed segmentation, Proceedings of the 8th International Symposium on Mathematical Morphology. 8 (2007) 265-276.

[23] R. O. Duda, P. E. Hart, D. G. Stork, Pattern Classification and Scene Analysis (2nd ed.), John Wiley \& Sons, 1995.

[24] X. Zhang, G. Thibault, E. Decencière, G. Quellec, G. Cazuguel, A. Erginay, P. Massin, A. Chabouis, Spatial normalization of eye fundus images, in: ISBI 2012: 9th IEEE International Symposium on Biomedical Imaging, 2012, Poster.

[25] S. Morales, V. Naranjo, A. Navea, M. Alcañiz, Computer-aided diagnosis software for hypertensive risk determination through fundus image processing, IEEE Journal of Biomedical and Health Informatics. 18 (2014) 1757-1763. doi:10.1109/JBHI.2014.2337960.

[26] A. Criminisi, P. Perez, K. Toyama, Region filling and object removal by exemplar-based image inpainting, IEEE Transactions on Image Processing. 13 (2004) 1200-1212. doi:10.1109/TIP.2004.833105.

[27] R. Bock, J. Meier, G. Michelson, L. G. Nyúl, J. Hornegger, Classifying glaucoma with image-based features from fundus photographs, Pattern Recognition: 29th DAGM Symposium, Proceedings. 29 (2007) 355-364. doi:10.1007/978-3-540-74936-3_36.

[28] F. Yin, J. Liu, D. W. K. Wong, N. M. Tan, C. Cheung, M. Baskaran, T. Aung, T. Y. Wong, Automated segmentation of optic disc and optic cup in fundus images for glaucoma diagnosis, 25th International Symposium on Computer-Based Medical Systems (CBMS). 25 (2012) 1-6. doi:10.1109/CBMS.2012.6266344.

[29] X. Yanwu, X. Dong, L. Stephen, L. Jiang, C. Jun, C. Cheung, A. Tin, W. T. Yin, Sliding window and regression based cup detection in digital 
fundus images for glaucoma diagnosis, International Conference on Medical Image Computing and Computer Assisted Intervention (MICCAI). 6893 (2011) 1-8. doi:10.1007/978-3-642-23626-6.

[30] Z. Zhang, F. S. Yin, J. Liu, W. K. Wong, N. M. Tan, B. H. Lee, J. Cheng, T. Y. Wong, ORIGA-light: An online retinal fundus image database for glaucoma analysis and research, Engineering in Medicine and Biology Society (EMBC), 32nd Annual International Conference of the IEEE. 32 (2010) 3065-3068. doi:10.1109/IEMBS.2010.5626137.

[31] Suman Sedai and Pallab Roy and Dwarikanath Mahapatra and Rahil Garnavi, Segmentation of Optic Disc and Optic Cup in Retinal Fundus Images Using Coupled Shape Regression, Proceedings of the Ophthalmic Medical Image Analysis Third International Workshop. 1 (2016) 1-8. doi:10.17077/omia.1040.

[32] A. Chakravarty, J. Sivaswamy, Coupled Sparse Dictionary for DepthBased Cup Segmentation from Single Color Fundus Image, Springer International Publishing, Cham, 2014, pp. 747-754. doi:10.1007/978-3319-10404-1_93. 